\title{
Correlation between plasma angiopoietin-1, angiopoietin-2 and matrix metalloproteinase-2 in coronary heart disease
}

\author{
Haoyu Wu ${ }^{1,2}$, Xiling Shou ${ }^{2}$, Lei Liang ${ }^{2}$, Congxia Wang ${ }^{1}$, Xiaowei Yao ${ }^{2}$, Gong Cheng ${ }^{2}$
}

\begin{abstract}
${ }^{1}$ Department of Cardiovascular Medicine, the Second Affiliated Hospital of Medical School, Xi'an Jiaotong University, Xi'an, Shaanxi, China

2Department of Cardiology, The People's Hospital of Shaanxi Province, Xi'an, Shaanxi, China
\end{abstract}

Submitted: 11 January 2015

Accepted: 2 February 2015

Arch Med Sci 2016; 12, 6: 1214-1219

DOI: 10.5114/aoms.2016.62909

Copyright $\odot 2016$ Termedia \& Banach

\section{Abstract}

Introduction: Angiopoietin-2 (Ang-2) plays a critical role in inducing tumor cell infiltration, and this invasive phenotype is caused by up-regulation of matrix metalloproteinase (MMP)-2. The relationship between Ang-2 and MMP-2 in atherosclerosis has not been reported yet. The aim is to measure the plasma concentrations of Ang-1, Ang- 2 and MMP- 2 and assess the correlation between the concentrations of these factors in coronary heart disease (CHD) patients.

Material and methods: The testing was done in a cross-sectional study. We prospectively enrolled 42 individuals with acute myocardial infarction, 42 individuals with unstable angina pectoris, 42 individuals with stable angina pectoris and 45 healthy control subjects. Concentrations of Ang-1, Ang-2 and MMP-2 were measured using the enzyme-linked immunosorbent assay (ELISA) method. Spearman's rank correlation was calculated to evaluate the relationships between MMP-2 and Ang-1, and MMP-2 and Ang-2 in patients with CHD.

Results: Patients with acute myocardial infarction and unstable angina pectoris had higher Ang-2 and MMP-2 levels compared with stable angina patients and healthy control subjects $(p<0.05)$, while concentrations of Ang-1 were not statistically different between the groups. Spearman's rank correlation showed that Ang-2 levels positively correlated with MMP-2 in patients with CHD $(r=0.679, p<0.001)$.

Conclusions: Plasma Ang- 2 and MMP-2 levels but not Ang- 1 levels were increased in patients with CHD. Ang- 1 correlated weakly with MMP-2, whereas the Ang-2 and MMP-2 correlation was strong in patients with CHD. Ang-2 may play a role in atherosclerosis, and have an interaction with MMP-2.

Key words: angiopoietin-1, angiopoietin-2, matrix metalloproteinase-2, coronary heart disease, correlation.

\section{Introduction}

Coronary heart disease (CHD) has been recognized as the leading cause of morbidity and mortality in developed as well as developing countries. In more than $90 \%$ of cases, the cause of myocardial ischemia is reduction of coronary blood flow owing to atherosclerotic coronary arterial obstruction [1]. Atherothrombosis is the development of atherosclerotic plaques

\author{
Corresponding author: \\ Congxia Wang PhD \\ Department of \\ Cardiovascular Medicine \\ the Second Affiliated \\ Hospital of Medical School \\ Xi'an Jiaotong University \\ 710004 Xi'an, Shaanxi, China \\ Phone: +86 2987679770 \\ E-mail: wcx0622@gmail.com
}


that eventually rupture, leading to superimposed thrombosis, vessel occlusion, and subsequent ischemic end organ damage, such as acute myocardial infarction (AMI) and stroke [2]. Because these ischemic events may be more dependent on the plaque composition than on the degree of stenosis, it is important to determine mechanisms that render the atherosclerotic plaque unstable and to search for biomarkers that identify patients at risk for plaque rupture [3].

Matrix metalloproteinases (MMPs) are a group of zinc-dependent endopeptidases with capacity to cleave several components of the extracellular matrix in the vessel wall, such as collagen, elastin, gelatins, and casein, potentially changing the atherosclerotic plaque composition [4, 5]. These changes then change the plaque into an unstable phenotype, prone to rupture and superimposed thrombosis. Matrix metalloproteinases- 2 is one of the potential targets for identification of vulnerable plaques [3].

Angiopoietin-1 (Ang-1) and angiopoietin-2 (Ang-2) are ligands of the tyrosine kinase receptor Tie-2 that contribute to blood vessel formation during angiogenesis. The roles of Ang-1 and Ang-2 during vascular development have been extensively investigated, as has their role in controlling the responsiveness of the endothelium to exogenous cytokines. Ang-1 and Ang- 2 also play roles in atherosclerosis. However, very little is known about the role of these vascular morphogenic molecules in the pathogenesis of atherosclerosis. Ang-2 may inhibit atherosclerosis by limiting low-density lipoprotein-cholesterol (LDL-C) oxidation via stimulation of nitric oxide production. In contrast, Ang-1 can promote monocyte and neutrophil migration [6].

Up-regulation of MMP-2, a member of the MMP family, has been found in glioma cell lines and in high-grade glioma specimens [7], and its activation has been linked to enhanced glioma invasion in several in vitro and in vivo model systems [8]. Ang-2 also plays a critical role in inducing tumor cell infiltration, and this invasive phenotype is caused by up-regulation of MMP-2 [9].

However, the relationship between Ang-2 and MMP-2 in atherosclerosis has not been reported yet. We therefore hypothesized that increasing levels of MMP-2 and Ang-2 are correlated and Ang-2 up-regulates the level of MMP-2 in patients with CHD. The aims of the present study were to measure the plasma concentrations of Ang-1, Ang-2 and MMP-2 and assess the correlation between the concentrations of these factors in CHD patients. The testing was done in a cross-sectional study. The experiment was performed with the understanding and consent of each subject.

\section{Material and methods}

\section{Patients}

We prospectively recruited patients presenting with their first AMI to our coronary care unit. The clinical diagnosis of AMI was based on the concurrence of prolonged (> $30 \mathrm{~min}$ ) chest pain or discomfort, elevated myocardial enzymes (total creatine kinase (CK) and CK-MB more than twice the upper normal limit and/or raised troponin I levels to at least the high-risk level) and electrocardiographic (ECG) changes (ST-T segment elevation or depression) occurring within $24 \mathrm{~h}$ of symptom onset. We also recruited patients presenting with unstable angina pectoris (UAP), defined as the presence of typical angina at rest or on minimum exertion associated with acute and transient ST-T segment ECG changes but with normal cardiac enzymes, including troponin levels. All patients received standard therapy, including aspirin, low-molecular-weight heparin, intravenous nitrates, a statin, $\beta$-blocker, and angiotensin-converting enzyme (ACE) inhibitor, when appropriate.

Baseline values of the acute patients were compared with those of age- and sex-matched patients with clinically stable angina pectoris (SAP) and healthy control subjects. Patients with SAP were recruited from those attending for elective day-case coronary angiography and found to have $\geq 1$ coronary stenoses ( $>50 \%$ severity) in major coronary arteries. In the same period, outpatients who underwent regular physical examinations were recruited as healthy control subjects. These individuals had no previous history of AMI, coronary bypass surgery, or percutaneous coronary intervention $(\mathrm{PCI})$. They were diagnosed free of CHD by their medical history of CHD or angiography, free of clear ischemic changes by electrocardiography and without chest pain symptoms.

Individuals with age $>75$ years, Killip class III or IV heart failure, significant valvular heart disease, previous history of AMI, atrial fibrillation, peripheral vascular disease, recent infections, chronic inflammatory diseases, connective tissue disease, chronic kidney, chronic hepatic disease and known history of neoplastic diseases, and people who are relatives were excluded from this study.

The local ethics committee approved the study, and all subjects gave informed consent.

\section{Blood sampling}

Venous blood samples were taken from the acute patients within $24 \mathrm{~h}$ of admission and $48 \mathrm{~h}$ thereafter between 6 and 7 a.m. while in a fasting condition. Within $30 \mathrm{~min}$ of collection, samples were centrifuged at $3000 \mathrm{rpm}$ for $20 \mathrm{~min}$, and stored at $-70^{\circ} \mathrm{C}$ until batch analysis. 


\section{Laboratory}

Ang-1, Ang-2 and MMP-2 were measured by commercial enzyme-linked immunosorbent assay (ELISA) as previously described in detail [10, 11]. All assays were performed in duplicate. Intra-assay and inter-assay coefficients of variation for all ELISA assays were $5 \%$ and $10 \%$, respectively.

\section{Coronary angiography}

All patients underwent elective coronary angiography according to the Judkins technique, and images were recorded offline for later analyses. Gensini scores were calculated to reflect the extent of coronary lesions [12].

\section{Statistical analysis}

Continuous variables were expressed as mean \pm standard deviation (SD). Comparisons between groups were performed by the Kruskal-Wallis or 1-way analysis of variance (ANOVA) with (after log transformation) Tukey's post hoc test set at a fixed $p<0.05$ or $p<0.01$. Categorical variables were expressed as the number (percent) and compared using the $\chi^{2}$ test. Pearson's correlation coefficients were calculated to evaluate the relationships between Ang-1, Ang-2, MMP-2 and several clinical variables. Spearman's rank correlation was calculated to evaluate the relationships between MMP-2 and Ang-1, and MMP-2 and Ang-2 in patients with CHD. A two-sided value of $p<0.05$ was considered statistically significant. All statistical analyses were performed with SPSS version 20 (SPSS Inc).

\section{Results}

The baseline clinical and demographic details are summarized in Table I. There were significant differences between the groups in terms of fasting plasma glucose (FPG), cholesterol and LDL-C.

Table II shows the cross-sectional results. There were no difference in Ang-1 levels between groups $(p=0.211)$. Overall, there were significant differences in the Ang- 2 data between groups $(p<0.001)$, and Tukey's post hoc test showed significant differences between $\mathrm{AMI}$ and the other 3 groups $(p<0.05)$. Ang- 2 was also raised in UAP compared with healthy control subjects and SAP. Similarly, MMP-2 levels ( $p<0.001$ overall) were significantly higher in AMI patients compared with UAP patients, SAP patients, and healthy control subjects, levels in patients with UAP were higher than in the control subjects and SAP, and levels in patients with SAP were higher than in the control subjects (all $p<0.05$ ). The Gensini score was significantly higher in AMI patients compared with UAP and SAP patients (all $p<$ 0.05). The Gensini score was also higher in UAP patients than SAP patients $(p<0.05)$.

A simple linear regression analysis was performed to test the correlation between Ang-1, Ang-2, MMP-2 and different covariates (Table III). The Gensini score positively correlated with Ang-2 and MMP-2 levels.

Table I. Baseline demographic and clinical characteristics of all subjects

\begin{tabular}{|c|c|c|c|c|c|}
\hline Parameter & AMI $(n=42)$ & UAP $(n=42)$ & $\operatorname{SAP}(n=42)$ & $\begin{array}{l}\text { Control subjects } \\
\quad(n=45)\end{array}$ & $P$-value \\
\hline Age [years] & $60.53 \pm 6.29$ & $59.31 \pm 8.34$ & $58.50 \pm 8.63$ & $59.66 \pm 10.86$ & 0.65 \\
\hline Male, $n(\%)$ & $28(66.67)$ & $26(61.90)$ & $24(57.14)$ & $27(60.00)$ & 0.78 \\
\hline $\mathrm{BMI}\left[\mathrm{kg} / \mathrm{m}^{2}\right]$ & $24.66 \pm 4.01$ & $23.76 \pm 2.04$ & $22.89 \pm 1.63$ & $23.68 \pm 2.44$ & 0.34 \\
\hline Active smokers, $n(\%)$ & $19(45.23)$ & $13(30.95)$ & $15(35.71)$ & $11(24.44)$ & 0.47 \\
\hline $\mathrm{SBP}[\mathrm{mm} \mathrm{Hg}]$ & $132.4 \pm 20.95$ & $130.62 \pm 17.24$ & $123.38 \pm 17.43$ & $131.87 \pm 11.33$ & 0.42 \\
\hline FPG $[\mathrm{mmol} / \mathrm{l}]$ & $7.66 \pm 3.18$ & $7.32 \pm 3.69$ & $6.21 \pm 2.27$ & $5.00 \pm 0.80$ & $<0.01$ \\
\hline Cholesterol [mmol/l] & $5.80 \pm 0.95$ & $5.28 \pm 1.29$ & $4.73 \pm 0.98$ & $4.63 \pm 0.60$ & 0.04 \\
\hline Triglyceride [mmol/l] & $1.86 \pm 0.59$ & $1.59 \pm 0.59$ & $1.50 \pm 0.69$ & $1.51 \pm 0.80$ & 0.47 \\
\hline LDL-C [mmol/l] & $3.63 \pm 0.69$ & $3.12 \pm 0.65$ & $3.43 \pm 0.63$ & $2.19 \pm 0.61$ & $<0.01$ \\
\hline Urea nitrogen [mmol/l] & $5.44 \pm 3.44$ & $5.97 \pm 1.17$ & $4.95 \pm 1.84$ & $4.97 \pm 1.69$ & 0.52 \\
\hline Creatinine $[\mu \mathrm{mol} / \mathrm{l}]$ & $80.64 \pm 36.77$ & $67.69 \pm 19.34$ & $64.66 \pm 17.14$ & $67.04 \pm 17.14$ & 0.24 \\
\hline Hypertension, $n$ (\%) & $19(45.24)$ & $23(54.76)$ & $18(42.86)$ & - & 0.63 \\
\hline Diabetes mellitus, $n(\%)$ & $11(26.19)$ & $8(19.05)$ & $6(14.29)$ & - & 0.08 \\
\hline
\end{tabular}

Data are shown as the mean \pm standard deviation and percentage (\%). BMI - body mass index, SBP - systolic blood pressure, FPG - fasting plasma glucose, LDL-C - low-density lipoprotein cholesterol, AMI - acute myocardial infarction, UAP - unstable angina pectoris, $S A P$ - stable angina pectoris. 
Table II. Baseline levels of research indexes among the 4 study groups

\begin{tabular}{|lccccc|}
\hline Parameter & AMI & UAP & SAP & Control subjects & $P$-value \\
\hline Ang-1 $[\mathrm{ng} / \mathrm{ml}]$ & $2.42 \pm 1.12$ & $2.37 \pm 0.82$ & $1.93 \pm 0.54$ & $1.92 \pm 0.85$ & 0.211 \\
\hline Ang-2 $[\mathrm{pg} / \mathrm{ml}]$ & $296.70 \pm 70.47^{\star \Delta} \triangle$ & $222.42 \pm 66.06^{\star \Delta}$ & $176.66 \pm 67.01$ & $133.18 \pm 35.55$ & $<0.001$ \\
\hline MMP-2 $[\mathrm{ng} / \mathrm{ml}]$ & $251 \pm 58.41^{\star \Delta} \triangle$ & $212.16 \pm 41.21^{\star \Delta}$ & $174.87 \pm 50.47^{\star}$ & $135.41 \pm 34.66$ & $<0.001$ \\
\hline Gensini score & $60.67 \pm 33.77^{\star} \triangle$ & $24.72 \pm 23.07^{\boldsymbol{\Delta}}$ & $22.90 \pm 17.09$ & $-<$ & $<.001$ \\
\hline
\end{tabular}

${ }^{\star} P<0.05$ vs. healthy control subjects, ${ }^{\Delta_{p}}<0.05$ vs. SAP, ${ }^{\triangle} p<0.05$ vs. UAP. Data are shown as mean \pm standard deviation. AMI - acute myocardial infarction, UAP - unstable angina pectoris, SAP - stable angina pectoris.

Table III. Correlations between Ang-1, Ang-2, MMP-2 and various parameters

\begin{tabular}{|c|c|c|c|c|c|c|}
\hline \multirow[t]{2}{*}{ Parameter } & \multicolumn{2}{|c|}{ Ang-1 } & \multicolumn{2}{|c|}{ Ang-2 } & \multicolumn{2}{|c|}{ MMP-2 } \\
\hline & $r$ & $p$ & $r$ & $p$ & $r$ & $p$ \\
\hline Age [years] & -0.046 & 0.724 & 0.084 & 0.516 & 0.087 & 0.499 \\
\hline $\mathrm{BMI}\left[\mathrm{kg} / \mathrm{m}^{2}\right]$ & 0.249 & 0.051 & 0.141 & 0.275 & 0.229 & 0.073 \\
\hline $\mathrm{SBP}[\mathrm{mm} \mathrm{Hg}]$ & -0.059 & 0.647 & 0.015 & 0.905 & -0.074 & 0.544 \\
\hline $\mathrm{FPG}[\mathrm{mmol} / \mathrm{l}]$ & 0.14 & 0.279 & 0.101 & 0.436 & -0.001 & 0.995 \\
\hline Cholesterol $[\mathrm{mmol} / \mathrm{l}]$ & -0.017 & 0.897 & 0.171 & 0.183 & 0.09 & 0.48 \\
\hline Triglyceride [mmol/l] & 0.014 & 0.913 & 0.121 & 0.349 & 0.165 & 0.201 \\
\hline LDL-C [mmol/l] & -0.033 & 0.802 & 0.260 & $<0.05$ & 0.176 & 0.170 \\
\hline Urea nitrogen [mmol/l] & 0.207 & 0.107 & 0.029 & 0.82 & -0.004 & 0.975 \\
\hline Creatinine $[\mu \mathrm{mol} / \mathrm{l}]$ & 0.036 & 0.781 & 0.03 & 0.815 & 0.207 & 0.107 \\
\hline Gensini score & 0.226 & 0.078 & 0.62 & $<0.001$ & 0.544 & $<0.001$ \\
\hline
\end{tabular}

Data are shown as mean \pm standard deviation and percentage (\%). BMI - body mass index, SBP - systolic blood pressure, FPG - fasting plasma glucose, $L D L-C$ - low-density lipoprotein cholesterol. Coefficients ( $r$ ) and p-values are calculated using the Pearson correlation model.

Overall, Ang-1 correlated weakly with MMP-2 $(r=0.245, p=0.055)$, whereas the Ang-2 and MMP-2 correlation was strong in patients with $\mathrm{CHD}(r=0.679, p<0.001$; Figure 1$)$.

\section{Discussion}

Matrix metalloproteinase are a family of zincand calcium-dependent proteolytic enzymes. The MMPs can degrade most extracellular matrix proteins [13]. This ability to modify the structural integrity of tissues is essential for certain aspects of normal physiology, including embryonic development, cell migration, wound healing, and tissue resorption. Matrix metalloproteinases' modification of integrins and activation of certain cell-signaling cascades also contribute to regulation of platelet function. However, the dysregulation or activation of MMP expression is a feature of numerous pathologic conditions, such as tumor metastasis, vascular and cardiac remodeling, and certain rheumatic conditions.

Several studies have shown that MMP-2 is involved in all stages of the atherosclerotic process, from the initial lesion to plaque rupture $[14,15]$. On one hand, MMPs' activity may contribute to the pathogenesis of atherosclerosis by facilitating migration of vascular smooth muscle cells through the internal elastic lamina into the intimal space, where they proliferate and contribute to plaque formation. On the other hand, MMPs' activity may diminish plaque volume by degrading extracellular matrix in the intima [16].

Of the four angiopoietins identified (Ang-1 to Ang-4), the best characterized are Ang-1 and Ang-2. Ang-1 and Ang-2 have both been identified

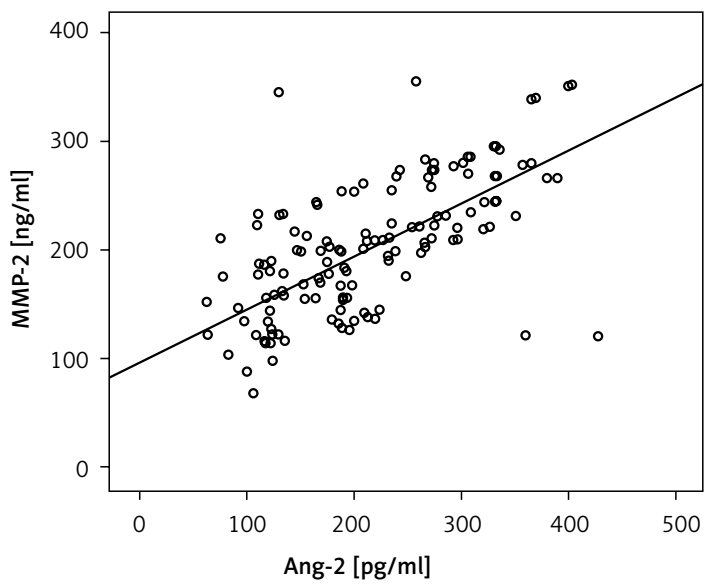

Figure 1. Correlation between plasma Ang-2 and MMP-2 levels in patients with CHD (Spearman's $r=0.679, p<0.001)$ 
as ligands for Tie-2, a receptor expressed on endothelial cells, and they play critical roles in angiogenesis [17]. Ang-1 promotes structural integrity of blood vessels. In contrast, Ang-2 is classically considered as a Tie-2 antagonist, counteracting the stabilizing effects of Ang-1. The local balance between these factors and the level of other angiogenic factors determine whether blood vessels grow, are maintained or become leaky and unstable. However, very little is known about the role of angiopoietins in atherogenesis. Ang-1 and Ang-2 appear to have diametrically opposite effects in the setting of cardiovascular disease. A recent study showed, contrary to expectations, that a single systemic administration of adenoviral Ang-2 to apoE ${ }^{-/-}$mice, fed a Western diet, reduced atherosclerotic lesion size and LDL-C oxidation in a nitric oxide synthase dependent manner [18]. Nykanen et al. [19] showed that adenoviral-mediated overexpression of Ang-1 protected against the development of arteriosclerosis in rat cardiac allografts, possibly by reducing the influx of leukocytes into the graft and thereby reducing inflammation and permeability. However, the same investigators later reported that long-term Ang-1 expression with adeno-associated virus-mediated intracoronary delivery failed to protect against the development of cardiac allograft arteriosclerosis [20]. From the above studies, we know that further investigation is required to clarify the mechanism underlying the effects of Ang-1 and Ang-2 in the development of atherosclerosis.

Recent studies have suggested that Ang-2 and MMP-2 play a critical role in human glioma invasion. Although the diverse roles of Ang-2 in tumor progression have been studied extensively, investigations of its functions have been primarily focused on its action on angiogenesis through a Tie-2-dependent pathway [21]. Ang-2 can also directly induce glioma cell invasion in the absence of detectable Tie-2 expression [9]. It has been reported that increased expression of MMP-2, MMP-9, and membrane type-1 MMP (MT1-MMP) is correlated with the invasive phenotype of glioma and other types of tumors. Up-regulation of Ang-2 and MMP-2 in tumor cells correlates with glioma invasion displayed by human gliomas [22]. Hu et al. [9] reported that Ang-2-stimulated glioma cell invasion results from the up-regulation of MMP-2 in tumor cells. Then they found that Ang-2 stimulated human glioma cell invasion by stimulating MMP-2 expression via the $\alpha v \beta_{1}$ integrin/FAK/p130Cas/ $\mathrm{ERK}_{1} / 2$ and $\mathrm{JNK}_{1}$ signaling pathway [23].

Matrix metalloproteinases and Ang-2 have been previously associated with the presence and progression of heart failure. Heart failure represents a complex multifactorial syndrome, characterized by crucial structural and functional abnormalities of the myocardium. Matrix metalloproteinases are associated with left ventricular dysfunction, adverse left ventricular remodeling and prognosis after acute myocardial infarction. There is a strong association between oxidative stress and MMPs in the pathophysiology of heart failure [24]. In hypertensive heart disease tissue inhibitors of matrix metalloproteinases (TIMPs) are increased and MMPs are decreased. The expression of MMP-2 and MMP-9 changes during hypertensive heart disease suggests that their activation contributes to cardiac remodeling [25]. Ang- 2 concentrations are significantly increased in patients with acute decompensated heart failure. Ang-2 might be used as a marker of disease severity, as a measure for clinical decompensation, and potentially as a promising target in patients with acute decompensated heart failure $[26,27]$.

However, there has been no study on the correlation and mechanism between Ang-2 and MMP-2 in atherosclerosis. The present study is the first to report the plasma concentrations of Ang-1, Ang- 2 and MMP- 2 in CHD patients, and assess the correlation between the concentrations of these factors. The results showed that plasma Ang-2 and MMP-2 levels but not Ang-1 levels were increased in patients with CHD. We found that, similar to MMP-2, the levels of Ang-2 changed in different types of CHD. Spearman's rank correlation showed that Ang-1 correlated weakly with MMP-2, whereas the Ang-2 and MMP-2 correlation was strong in patients with CHD. These results suggest that Ang-2 plays a role in atherosclerosis, and might have an interaction with MMP-2.

There are many risk factors for CHD, such as hypertension, increased body mass index (BMI), increased abdominal girth, dyslipidemia, diabetes mellitus, etc. [28]. In this study, we also found that there are significant differences between the groups in terms of FPG, cholesterol and LDL-C that increase with the severity of coronary artery disease.

Given the evidence discussed, our findings of raised Ang- 2 and MMP-2 and their positive correlation suggest that MMP-2 might play a role in Ang-2-induced atherosclerosis, although we have no direct evidence that Ang-2 up-regulates the level of MMP-2 in patients with CHD. Given the deep research on Ang-2 and MMP-2 correlation and mechanism in cancer, we have enough reasons to suppose this relationship exists in atherosclerosis, and we need a future study to confirm this relationship. Indeed, our findings provide further insight into the pathophysiology of the disease and raise very important research questions that will be the subject of future study as we seek to understand the role of angiogenesis in atherosclerosis. In the future, studies of these factors, using larger sample sizes and histological experiments in vivo and in vitro, should be performed to 
further elucidate the relationship between Ang-2 and MMP-2 in atherosclerosis.

However, our study has some limitations. First and most importantly, this is only a single-center study, with cross-sectional design and a small number of patients. In this setting, the results of the study should be interpreted with caution. However, in our opinion, the results of this study strongly indicate the correlation between Ang-2 and MMP-2 in atherosclerosis. Therefore, larger studies with a higher number of patients should be performed to investigate this issue. Furthermore, we need future studies to confirm this relationship between Ang-2 and MMP-2 in atherosclerosis.

In conclusion, the correlation between Ang-2 and MMP-2 is strong in patients with CHD, suggesting that Ang-2 may play a role in atherosclerosis, and have an interaction with MMP-2. Further studies are needed to confirm this result.

\section{Acknowledgments}

This study was supported by National Natural Science, Foundation of China (81273878).

\section{Conflict of interest}

The authors declare no conflict of interest.

\section{References}

1. Mallika V, Goswami B, Rajappa M. Atherosclerosis pathophysiology and the role of novel risk factors: a clinicobiochemical perspective. Angiology 2007; 58: 513-22.

2. Sakakura K, Nakano M, Otsuka F, Ladich E, Kolodgie FD, Virmani R. Pathophysiology of atherosclerosis plaque progression. Heart Lung Circulation 2013; 22: 399-411.

3. Ketelhuth DF, Back $M$. The role of matrix metalloproteinases in atherothrombosis. Curr Atheroscler Rep 2011; 13: 162-9.

4. Back M, Ketelhuth DF, Agewall S. Matrix metalloproteinases in atherothrombosis. Prog Cardiovasc Dis 2010; 52: 410-28.

5. Rysz J, Banach M, Stolarek RA, et al. Serum matrix metalloproteinases MMP-2 and MMP-9 and metalloproteinase tissue inhibitors TIMP-1 and TIMP-2 in diabetic nephropathy. J Nephrol 2007; 20: 444-52.

6. Ahmed A, Fujisawa T. Multiple roles of angiopoietins in atherogenesis. Curr Opin Lipidol 2011; 22: 380-5.

7. Yong VW, Power C, Forsyth P, Edwards DR. Metalloproteinases in biology and pathology of the nervous system. Nat Rev Neurosci 2001; 2: 502-11.

8. Chintala SK, Tonn JC, Rao JS. Matrix metalloproteinases and their biological function in human gliomas. Int J Dev Neurosci 1999; 17: 495-502.

9. Hu B, Guo P, Fang Q, et al. Angiopoietin-2 induces human glioma invasion through the activation of matrix metalloprotease-2. Proc Natl Acad Sci USA 2003; 100: 8904-9.

10. Melen-Mucha G, Niedziela A, Mucha S, et al. Elevated peripheral blood plasma concentrations of tie-2 and angiopoietin 2 in patients with neuroendocrine tumors. Int J Mol Sci 2012; 13: 1444-60.
11. Vasaturo F, Solai F, Malacrino C, et al. Plasma levels of matrix metalloproteinases 2 and 9 correlate with histological grade in breast cancer patients. Oncol Lett 2013; 5: 316-20

12. Gensini GG. A more meaningful scoring system for determining the severity of coronary heart disease. Am J Cardiol 1983; 51: 606.

13. Hadler-Olsen E, Winberg JO, Uhlin-Hansen L. Matrix metalloproteinases in cancer: their value as diagnostic and prognostic markers and therapeutic targets. Tumour Biol 2013; 34: 2041-51.

14. Kai H, Ikeda H, Yasukawa $\mathrm{H}$, et al. Peripheral blood levels of matrix metalloproteases- 2 and -9 are elevated in patients with acute coronary syndromes. J Am Coll Cardiol 1998; 32: 368-72.

15. Galis ZS, Sukhova GK, Lark MW, Libby P. Increased expression of matrix metalloproteinases and matrix degrading activity in vulnerable regions of human atherosclerotic plaques. J Clin Invest 1994; 94: 2493-503.

16. Kampoli AM, Tousoulis D, Papageorgiou N, et al. Matrix metalloproteinases in acute coronary syndromes: current perspectives. Curr Top Med Chem 2012; 12: 1192-205.

17. Engin H, Ustundag Y, Ozel Tekin I, Gokmen A. Plasma concentrations of ang- 1 , ang- 2 and tie- 2 in gastric cancer. Eur Cytokine Netw 2012; 23: 21-4.

18. Ahmed A, Fujisawa T, Niu XL, et al. Angiopoietin-2 confers atheroprotection in apoe-/- mice by inhibiting Idl oxidation via nitric oxide. Circ Res 2009; 104: 1333-6.

19. Nykanen Al, Krebs R, Saaristo A, et al. Angiopoietin-1 protects against the development of cardiac allograft arteriosclerosis. Circulation 2003; 107: 1308-14.

20. Nykanen Al, Pajusola K, Krebs R, et al. Common protective and diverse smooth muscle cell effects of aav-mediated angiopoietin- 1 and -2 expression in rat cardiac allograft vasculopathy. Circ Res 2006; 98: 1373-80.

21. Li Q, Xu B, Fu L, Hao XS. Correlation of four vascular specific growth factors with carcinogenesis and portal vein tumor thrombus formation in human hepatocellular carcinoma. J Exp Clin Cancer Res 2006; 25: 403-9.

22. Guo P, Imanishi Y, Cackowski FC, et al. Up-regulation of angiopoietin-2, matrix metalloprotease-2, membrane type 1 metalloprotease, and laminin 5 gamma 2 correlates with the invasiveness of human glioma. Am J Pathol 2005; 166: 877-90.

23. Hu B, Jarzynka MJ, Guo P, Imanishi Y, Schlaepfer DD, Cheng SY. Angiopoietin 2 induces glioma cell invasion by stimulating matrix metalloprotease 2 expression through the alphavbeta1 integrin and focal adhesion kinase signaling pathway. Cancer Res 2006; 66: 775-83.

24. Tousoulis D, Kampoli AM, Papageorgiou N, et al. Matrix metallopropteinases in heart failure. Curr Top Med Chem 2012; 12: 1181-91.

25. Gluba A, Bielecka-Dabrowa A, Mikhailidis DP, et al. An update on biomarkers of heart failure in hypertensive patients. J Hypertens 2012; 30: 1681-9.

26. Pöss J, Ukena C, Kindermann I, et al. Angiopoietin-2 and outcome in patients with acute decompensated heart failure. Clin Res Cardiol 2015; 104: 380-7.

27. Eleuteri E, Di Stefano A, Tarro Genta F, et al. Stepwise increase of angiopoietin-2 serum levels is related to haemodynamic and functional impairment in stable chronic heart failure. Eur J Cardiovasc Prev Rehabil 2011; 18: 607-14.

28. Banach M, Serban C, Aronow WS, et al. Lipid, blood pressure and kidney update 2013. Int Urol Nephrol 2014; 46: 947-61. 